\title{
Dual-Collector Lateral Bipolar Magnetotransistor: Negative Sensitivity and Galvanomagnetic Effects
}

\author{
R.D. Tikhonov*
}

SMC "Technological Centre" MIEE, Zelenograd, Moscow 124 482, Russia

\begin{abstract}
The results of measurement of the dual-collector lateral bipolar magnetotransistor, generated in a uniformly doped substrate or in a diffused well were compared and it was established that the collector voltage difference under the influence of a magnetic field has a different sign. The effect is interpreted using the concept of the magnetic sensitivity sign. By means of device-technological simulation we investigated the distribution of charge carriers, current density and recombination speed in a magnetotransistor generated in a well with external connection of substrate and well contacts. It is shown that at a planar arrangement of substrate contacts is responsible for the volumetric concentration-recombination mechanism of negative relative magnetic sensitivity due to Galvanomagnetic effects.
\end{abstract}

Keywords: Bipolar magnetotransistor, mechanism of sensitivity, galvanomagnetic effects.

\section{INTRODUCTION}

The effect of electric and magnetic fields on chaotically moving charge carriers in a semiconductor with uniform distribution of the impurity results in a drift of carriers within the free run in the direction of the electric field action and curving of the carriers' trajectory under the Lorentz force transverse to the electric field action. The electric field creates a current, and with an active magnetic field, there are two effects: The longitudinal Galvanomagnetic Gauss effect - change in the semiconductor resistance in the magnetic field, supplement with a cross effect resulting in the evolvement of the cross Galvanomagnetic effect - the Hall Voltage between the lateral surfaces of the sample [1]. The change of resistance is defined as a change of mobility at each point of the semiconductor or as a current line deflection increasing the effective length. The Hall effect is determined by accumulation of charges of different signs on the opposite lateral surfaces of the sample.

In the intrinsic semiconductor, two types of charge carriers, electrons and holes, in the electric field drift towards each other and are deflected by the magnetic field in the same direction. The electron-hole plasma concentration increases on one lateral surface and decreases on the other, which determines an increase of the Gauss effect and a decrease of the Hall effect. This is accompanied by the galvanomagnetic-recombination effect [2-4]. The effective gain is achieved with the processing of the sample surfaces in order to speed up recombination. In magnetodiodes, the second type of carrier is entered by injecting minority charge carriers [5] in the base area comparable with the diffusion length, which allows increasing magnetic sensitivity by many times.

The vertical bipolar magnetotransistor (BMT) is based on the effect of the deflection under a Lorentz force in a magnetic field directed along the surface of a crystal, a flow of charge carriers injected from the emitter in the transistor

*Address correspondence to this author at the SMC "Technological Centre" MIEE, Zelenograd, Moscow 124 482, Russia, E-mail: r.tikhonov@ @cen.ru base, and redistribution of the flow between the split collector [6].

The planar BMT [5] uses the flow of charge carriers injected along the crystal surface. The magnetic field directed perpendicularly to the surface deflects the flow towards one of the two collectors located on the surface. Extraction of carriers by collectors is equivalent to the high recombination speed on the surface. The deflection effect results in redistribution of the flow of carriers between collectors and changes the effective length of the current line.

In the lateral BMT [7], the emitter is located between the collectors on the crystal surface. The magnetic field is directed along the crystal surface. The injected charge carriers under the field of action are deflected on one side of the emitter towards the surface, which shortens current lines, reduces the extent of recombination, and simultaneously forces up recombination on the surface. On the other side of the emitter, current lines move away from the crystal surface and become longer, recombination grows in volume, and decreases on the surface. With the level of injection being high, the magnetoconcentration effect is observed [8], connected with modulation in the magnetic field of the bipolar magnetotransistor base resistance by the injected minority carriers and the majority carriers compensating for their charge. The magnetoconcentration effect results in the formation of highly localized current filaments [9].

In the lateral BMT in CMOS technology [10] the base is the diffusion well. In the lateral BMT with suppressed sidewall injection between the emitter and the collector, the base-well is doped or oxide ring, which limits the flow of injected charge carriers along the surface and reduces the influence of surface recombination[11-13].

The application of a two-dimensional simulation has shown that in the vertical BMT [14], the basic hole stream passes in the passive base as far as the emitter edges. Injection from the emitter occurs mainly from the edge areas. Two streams of injected electrons pass perpendicularly to the substrate surface from the emitter through the active base 
into the low-doped collector and further to the collector contacts. Distribution of the base hole current changes in the magnetic field directed along the emitter. The hole current drifts from one edge of the emitter and is pressed close to the other edge. The hole current change results in a change of electron injection from the emitter and a change of collector currents. In the collector, the magnetic field changes the bend of current lines.

For the lateral BMT with suppressed lateral injection [14], the influence of the magnetic field on the drift current of the majority carriers in the base is considered determinant for the occurrence of sensitivity. This also changes the distributed base resistance, i.e. the magnetoconcentration effect occurs.

\section{SIGN MAGNETOSENSITIVITY SEMICONDUCTOR DEVICES}

OF

The influence of magnetic field on semiconductor devices is defined by the interaction of the current and magnetic field and thereby occurrences of the Lorentz force $\boldsymbol{F}$ which acts on a current as the vector product of current density $\boldsymbol{J}$ and magnetic induction $\boldsymbol{B}$.

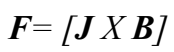

Change of a current under the influence of a magnetic field allows these devices to be known as currentmagnetic converters. Sensitivity of a current of electronic devices to a magnetic field is calculated based on the derivative dependence of current $\boldsymbol{I}(\boldsymbol{B})$, a current proceeding through the device from a magnetic induction $\boldsymbol{B}$. The increase in a current under the influence of a magnetic field gives a positive sign on a derivative $\boldsymbol{d I}(\boldsymbol{B}) / \boldsymbol{d} \boldsymbol{B}>0$. Reduction of a current in a magnetic field and accordingly $\boldsymbol{d I}(\boldsymbol{B}) / \boldsymbol{d} \boldsymbol{B}<\boldsymbol{0}$ can be treated as a negative sign on a derivative as negative sensitivity [15].

Resistance magnetoresistors of the rectangular and cylindrical form raises in a magnetic field [1]. Under the action of force of Lorentz direct lines of density of a current turn to arches and the effective length of the device (Fig. 1a) increases. At the set voltage on the magnetoresistor, the initial density of a current $\boldsymbol{j}(\mathbf{0})$ decreases [16] under the formula

$j(B)=j(0) /\left(1+\mu^{2} B^{2}\right)$

where $\mu$ - - geometrical magnetoresistor mobility.

Sensitivity $\boldsymbol{d I}(\boldsymbol{B}) / \boldsymbol{d} \boldsymbol{B}$ has a negative sign.

In the magnetodiode, electrons are injected from $\mathrm{N}+$ end of the emitter in the i-area with a small concentration of carriers and gather on the $\mathrm{P}+$ contact. [17]. Under the action of force of Lorentz direct lines of density of an electronic current turn to arches and the effective length of i-area (Fig. 1b) increases. In comparison with the magnetoresistor, the effective recombination of the injected carriers in the i-area and on a surface of its lateral faces is added. In the formula of a direct current of the diode, the multiplier $c$, describing a parity of length of i-area, and diffusion lengths is added.

\section{$I=I c(\exp (q U / c k T-1)$}

The factor $c$ is connected with mobility electrons $\boldsymbol{\mu}_{\boldsymbol{n}^{-}}$and holes $\boldsymbol{\mu}_{p}$, the length $\mathrm{i}$ - areas $\boldsymbol{d}$, and lifetime of the injected carriers of a charge $\tau_{n}$ under the formula

$c=2(b+c h d / L) /(b+1)$ where $b=\mu_{n} / \mu_{p}, L=\left(D_{n} \tau_{n}\right)^{1 / 2}[2 b /(b+1)]^{1 / 2}$ - effective length diffusion displacement, $\boldsymbol{D}_{\boldsymbol{n}}$ - factor of diffusion.

Lengthening of current lines is accompanied by an increase in $c$. The diode current on the increased line length of the current decreases due to recombination and sensitivity grows. In a magnetic field, carriers nestle on a surface and superficial recombination brings the contribution to current reduction and an increase in sensitivity. At the set voltage and a magnetic induction perpendicular to a direction of a current, the current magnetodiode decreases and therefore has negative sensitivity.
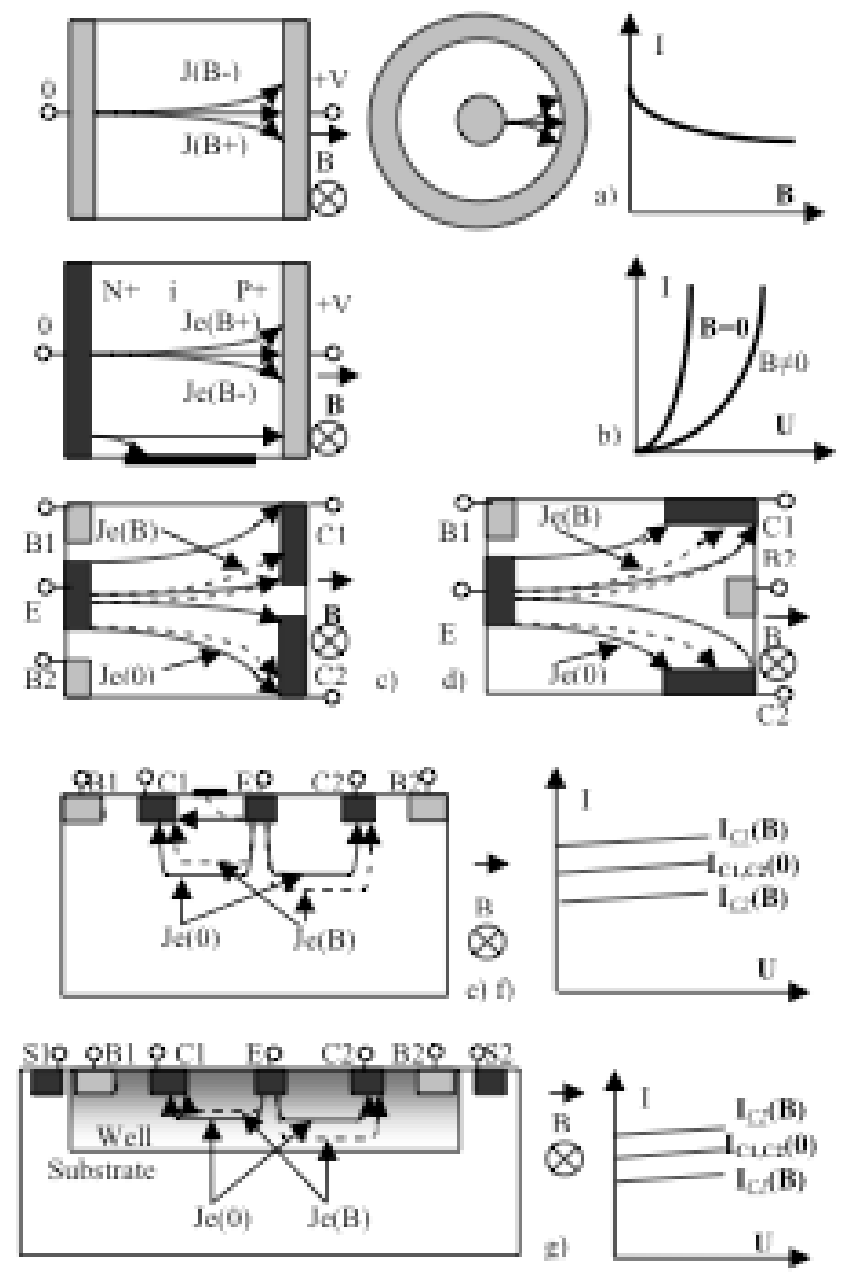

Fig. (1). Lines of a current and change of a current in a magnetic field in structures: a) magnetoresistor, b) magnetodiode, c) d) e) f) vertical, planar and lateral two-collector magnetotransistors, g) lateral two-collector magnetotransistor, generated in diffusion well.

In the vertical two-collector magnetotansistor (the Fig. 1c), electrons are injected from the $\mathrm{N}+$ end of the emitter in the i-area of the base and gather for two $\mathrm{N}+$ collectors [6]. Except for the three listed above factors in the transistor, action of a magnetic field leads to the change in distribution of the injected electrons between collectors C1 and C2. More electrons come to collector $\mathrm{C} 1$, its current increases, $\boldsymbol{d} \boldsymbol{I}_{\boldsymbol{C I}}(\boldsymbol{B}) / \boldsymbol{d} \boldsymbol{B}>0$, and sensitivity is positive. Less electrons come to collector $\mathrm{C} 2$, its current decreases, $\boldsymbol{d I _ { C I }}(\boldsymbol{B}) / \boldsymbol{d B}>0$, and sensitivity is negative. At a reverse direction of a magnetic induction, the sign on the sensitivity collectors changes. 
Two collector magnetotansistors are sensitive to the direction of a magnetic induction.

In the planar two-collector magnetotansistor (the Fig. 1d), electrons are injected from the $\mathrm{N}+$ end of the emitter in the p-area of the base and gather for two $\mathrm{N}+$ the collectors located on the parties from an axis, connecting the emitter and contact to the base $[5,18,19]$. Except for the four listed above factors in the transistor, the action of a magnetic field leads to the change in distribution of streams injected electrons to collectors $\mathrm{C} 1$ and $\mathrm{C} 2$ due to the change in the distribution of lines of a current and their effective length along the collectors. More electrons come to collector C1,its current increases, $\boldsymbol{d I}_{\boldsymbol{C} \boldsymbol{I}}(\boldsymbol{B}) / \boldsymbol{d} \boldsymbol{B}>0$, and sensitivity is positive. Less electrons come to collector $\mathrm{C} 2$, its current decreases, $\boldsymbol{d} \boldsymbol{I}_{\boldsymbol{C} 2}(\boldsymbol{B}) / \boldsymbol{d} \boldsymbol{B}<0$, and sensitivity is negative.

In the lateral two-collector magnetotansistor (the Fig. 1e), electrons are injected from the $\mathrm{N}+$ end of the emitter in the $\mathrm{P}$-a substrate which forms the base [7]. Two $\mathrm{N}+$ collectors are located on the surface of a substrate on the parties from the emitter. Contacts to the base are located on a surface of a substrate behind collectors. Except for the five listed above factors in the transistor, the action of a magnetic field leads to a change in the distribution of streams injected electrons to collectors $\mathrm{C} 1$ and $\mathrm{C} 2$ due to the change in position of the lines of a current concerning a surface. Lines of a current from collector $\mathrm{C} 1$ nestle on a surface and leave from a surface from collector $\mathrm{C} 2$. At the uniform distribution of an impurity in a substrate, this factor changes the contribution of superficial recombination in the currents of two collectors. If the role of superficial recombination is insignificant, more electrons come to collector $\mathrm{C} 1$, its current increases, $\boldsymbol{d} \boldsymbol{I}_{\boldsymbol{C I}}(\boldsymbol{B}) / \boldsymbol{d B}>0$, and sensitivity is positive. Less electrons come to collector $\mathrm{C} 2$, its current decreases, $\boldsymbol{d I _ { C 2 }}(\boldsymbol{B}) / \boldsymbol{d B}<0$, and sensitivity is negative. For the three types of transistors described, characteristic change of a current of collectors in a magnetic field is presented in Fig. 1f. At the big distance along a surface from the emitter up to collectors surface recombination gives the big contribution to sensitivity and change of a sign on sensitivity change of a level of injection $[20,21]$ is observed.

A lateral two-collector magnetotansistor (the Fig. 1g) is formed in the field of which refers to a diffusion well with non-uniform distribution of an impurity [10,22]. About a surface concentration of high impurity, it is equal in concentration of an impurity in a substrate at some depth. In this place, a pn-junction is formed in the field of volumetric charge and there are no carriers of a current. At a nonuniform distribution of an impurity in a substrate, the factor of change of the position of lines of a current concerning a surface results from collector $\mathrm{C} 1$ in moving lines of a current from the area with the minimal concentration of an impurity aside from the big concentration of an impurity. From collector $\mathrm{C} 2$, lines of a current move in a magnetic field in the direction of the area with a minimal concentration. Because of strengthening recombination, less electrons come in collector $\mathrm{C} 1$, its current decreases, $\boldsymbol{d} \boldsymbol{I}_{\boldsymbol{C} \boldsymbol{I}}(\boldsymbol{B}) / \boldsymbol{d} \boldsymbol{B}<0$, and sensitivity is negative. More electrons come to collector $\mathrm{C} 2$, its current increases, $\boldsymbol{d} \boldsymbol{I}_{\boldsymbol{C} 2}(\boldsymbol{B}) / \boldsymbol{d} \boldsymbol{B}>0$, and sensitivity is positive. Change of a current of collectors in a magnetic field is presented in a Fig. 1g and is opposite in comparison from a Fig. 1f.
This fact has been found out experimentally [23] and has demanded a detailed explanation [21,24-31]. In a given clause, it is carried out by means of device-technological modeling research BMT in the diffusion well at connection of contacts to a substrate on top or on the bottom surface of a substrate.

\section{EXPERIMENTAL DEMONSTRATION}

In the given work investigated, lateral double-collector bipolar magnetotransistors are generated in a substrate (BMT) and in a well (BMTW).

Samples of circuits were placed in an electromagnetic noise-screening box with the battery power supply of a constant voltage of 1.5-9 $\mathrm{V}$ with a changeable number of batteries [32]. The circuit of activation is shown in Fig. (2) for the BMTW. The circuits of activation at BMT measurements were characterized by the absence of contact connection of a well and a substrate. Face values of resistors had the following values: for $\mathrm{BMT}-\mathrm{Rb}=39.6 \mathrm{kOhm}, \mathrm{Rc}=27.38 \mathrm{kOhm}$, for $\mathrm{BMTW}-\mathrm{Rb}=4.738 \mathrm{kOhm}, \mathrm{Rc}=27.38 \mathrm{kOhm}$.

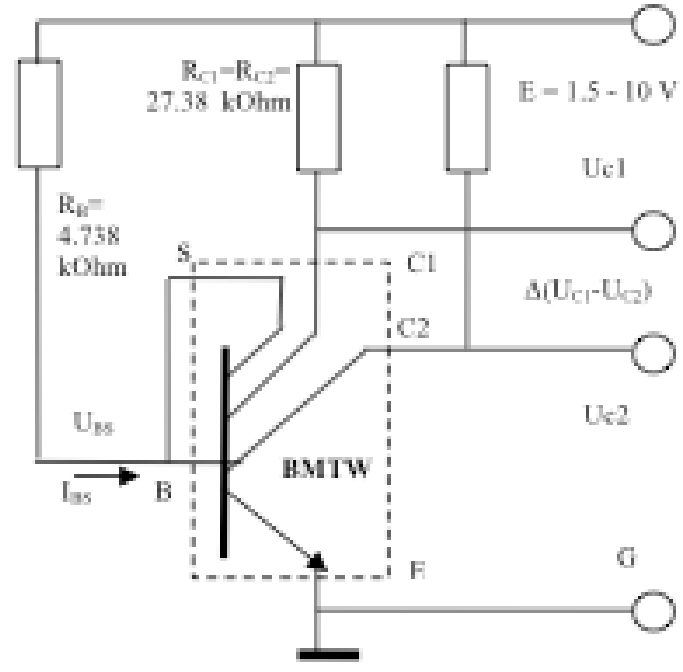

Fig. (2). Electric circuit for measurement dual-collector $\mathrm{C} 1, \mathrm{C} 2$ lateral magnetotansistor BMTW with base - well B, substrate S, emitter E.

Table 1 gives BMT electric parameters. With the growth of supply voltage, the current of a collector $I_{B}$ increases and a voltage $\mathrm{U}_{\mathrm{C}}$ on a collector increase.

Table 1. Dependence of Electric Parameters BMT on a Supply Voltage

\begin{tabular}{|c|c|c|c|}
\hline $\mathbf{E}, \mathbf{V}$ & $\mathbf{I}_{\mathbf{B}}, \boldsymbol{\mu} \mathbf{A}$ & $\mathbf{I}_{\mathbf{C}}, \boldsymbol{\mu} \mathbf{A}$ & $\mathbf{U}_{\mathbf{C}}, \mathbf{V}$ \\
\hline \hline 1.5 & 18 & 21 & 1.49 \\
\hline 3 & 55 & 44 & 1.77 \\
\hline 6 & 130 & 124 & 2.53 \\
\hline 9 & 205 & 220 & 3.84 \\
\hline
\end{tabular}

Transistors BMTW were made with the same topology as a BMT in a substrate. The type of conductivity of a substrate had been changed and a P-type diffusion well with superficial concentration two orders of higher than in a substrate for 
BMT was created. Calculations of the structure BMTW gave identical experimental values.

The difference of voltages between collectors in a field of a constant magnet with an induction of $60 \mathrm{mT}$ detecting sensitivity of BMT, is presented on Fig. (3) depending on a supply voltage.

$\Delta U_{C}=\left[U_{C I}(B)-U_{C 2}(B)\right]-\left[U_{C I}(0)+U_{C 2}(0)\right]$

This difference increases with the growth of the supply voltage and gives the value of sensitivity at $\mathrm{E}=9 \mathrm{~V} \boldsymbol{S}_{U c}=$ $1.8 \mathrm{~V} / \mathrm{T}$ for BMT in substrate.

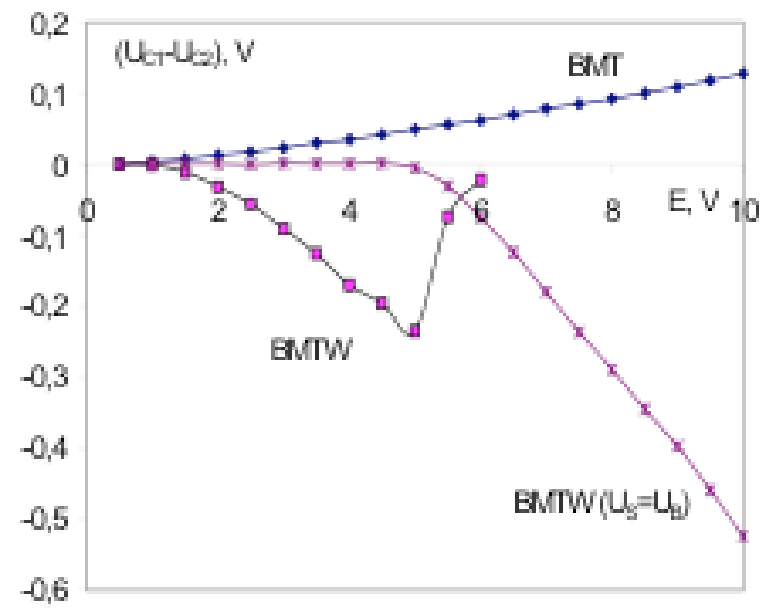

Fig. (3). Dependence of voltage differences of BMT and BMTW collectors on supply voltage on Fig. (2).

The maximal difference makes $\boldsymbol{\Delta} \boldsymbol{U}_{\boldsymbol{C}}=128 \mathrm{mV}$ and corresponds to absolute sensitivity voltage $S_{U c}=2,1 \mathrm{~V} / \mathrm{T}$.

$S_{U c}=\Delta U_{C} / B$

The difference of collectors voltages BMTW on Fig. (3) has a negative sign in comparison with BMT and the maximal sensitivity value of BMTW with the floating potential of a substrate was observed at $\mathrm{E}=5 \mathrm{~V}$. At a greater voltage, the transistor passed in saturation. The value of voltage sensitivity makes $\boldsymbol{S}_{U c}=-4 \mathrm{~V} / \mathrm{T}$ at $\boldsymbol{\Delta} \boldsymbol{U}_{\boldsymbol{C}}=-235 \mathrm{mV}$.

When connecting contacts to a substrate and to a basewell BMTW, the growth of the voltage difference begins with the operation of threshold voltage $5 \mathrm{~V}$ and continues to grow at the maximum supply voltage. The value of sensitivity reaches up to $S_{U c}=-9 \mathrm{~V} / \mathrm{T}$ at $\Delta \boldsymbol{U}_{C}=-524 \mathrm{mV}$.
Currents and BMTW sensitivity are resulted in Table $\mathbf{2}$ for an operating mode with the maximal sensitivity.

The maximal size of the BMTW sensitivity in the connection of contacts to a substrate and base contact increases by approximately two times. The emitter current doubles simultaneously. The tapping of injected electrons to contacts to substrate reduces the collector current that allows having greater emitter current before the collectors are saturated. The emitter current under influence of the Lorentz force changes in collector current and increases sensitivity. It is possible to consider, that at the chosen geometry, BMT sensitivity is practically defined by the value of the emitter current and the considered designs allow increasing emitter current until the transistor will be saturated in the collector current.

Change of voltage on BMT collectors on a substrate corresponds to a deflection of the injected emitter electrons under the Lorentz force to the side of the second collector. The current grows in the second collector and decreases in the first. The power failure on resistance of loading of the first collector falls and the voltage on a collector grows. Voltage drop on load resistance of the second collector grows and the voltage on a collector decreases. Sensitivity is defined as positive.

Change of a voltage on BMTW collectors has an opposite sign. With an absolute deviation of injected emitter electrons under the Lorentz force, there is a growth in the first collector current in the same direction and reduction of the second collector current. Sensitivity is negative.

The effect of sensitivity sign change is defined by distribution of current lines of the injected carriers in a well and recombination of the injected carriers and the basic carriers in base. Basically, the current proceeds close to the pn-junction of the well-substrate, where impurity concentration is lower due to diffusion distributions. In a magnetic field, the deflection of current lines leads to their displacement in the area of a well.

When the current lines approach the surface, the current of a collector should increase. However, current lines get to the area of a well with higher concentration of impurity that leads to recombination strengthening and to reduction of the collector current. There is the opposite situation on other collector. Current lines deflected from a surface to a wellsubstrate junction, where impurity concentration is lower. Recombination reduction and growth of a collector current take place.

Table 2. Results of Measurement of Sensitivity BMT

\begin{tabular}{|c|c|c|c|c|c|c|}
\hline № (Fig 3) & Type BMT & $\mathbf{U}_{\mathbf{C} \mathbf{1}-\mathbf{U}_{\mathbf{C} 2} \mathbf{m V}}$ & $\mathbf{I}_{\mathbf{C}}, \boldsymbol{\mu} \mathbf{A}$ & $\mathbf{I}_{\mathbf{B}}(\mathbf{1 , 2}) \mathbf{I}_{\mathbf{B S}}(\mathbf{3}), \boldsymbol{\mu} \mathbf{A}$ & $\mathbf{I}_{\mathbf{E}}, \mathbf{m A}$ & $\mathbf{S}_{\mathrm{Amax}}, \mathbf{V} / \mathbf{T}$ \\
\hline \hline 1 & BMT & 128 & 152 & 235 & 0.54 & 2.1 \\
\hline 3 & BMTW & -235 & 190 & 2000 & -4 \\
\hline & $\begin{array}{c}\text { BMTW, in } \\
\text { connection } \\
\text { to contacts } \\
\text { to base and } \\
\text { substrate }\end{array}$ & -524 & 280 & 2.56 & -8.7 \\
\hline
\end{tabular}


The increase in an identical current of collectors in two structures BMTW occurs at a different current of the emitter and leads to increase of sensitivity in a structure with great value of the emitter current. The division principle of an injection emitter current on components of measuring the collector current and the third pn-junction collector base-well allows it to reach a high value of BMTW sensitivity.

BMTW has a high level of sensitivity at a small collector current, which allows using greater face values of resistance of collector load resistance. In a mode of maximal sensitivity, the BMTW has the better detectability than the BMT.

Despite of obvious to all those who is engaged in bipolar transistors, dependence of factor of carry of the injected carriers of a charge on speed recombination in base in one of known works [33] recombination was not considered at the analysis of the mechanism of sensitivity BMT.

\section{TO OPTIMIZE THE STRUCTURE BMT}

The research of dependence of sensitivity on technological parameters for the well creation (doping doze and distances from emitter to the operating collectors) for BMTW was carried out [34].

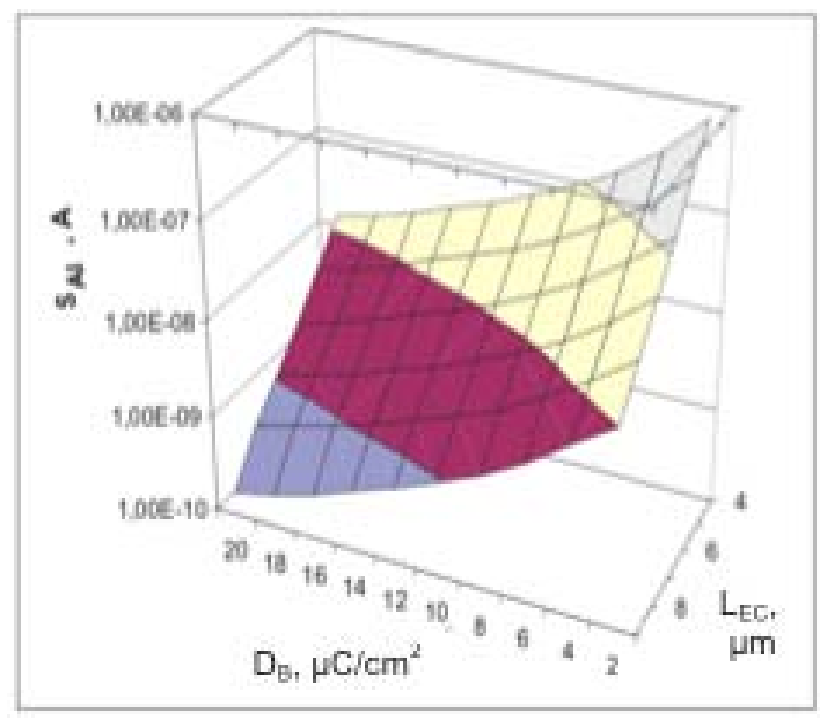

Fig. (4). Dependence of the absolute current magnetosensitivity on the bias $0.72 \mathrm{~V}$ between the emitter and contacts to the well-base and the substrate in the bipolar dual-collector lateral magnetotransistor (BMT) about the boros dose $2-20 \mu \mathrm{C} / \mathrm{cm}^{2}$ and the emittercollector distance $4-9 \mu \mathrm{m}$.

The three-dimensional diagram of absolute current sensitivity $\mathrm{S}_{\mathrm{AI}}$ dependence on parameters of the well is represented on Fig. (4). The value of magnetic induction is 1 $\mathrm{T}$ and is directed along the long side of the emitter. The maximum values of absolute current sensitivity $(0.8 \mu \mathrm{A} / \mathrm{T})$ can be observed given the bias voltage of $0.72 \mathrm{~V}$, the collector voltage $\mathrm{U}_{\mathrm{C} 1}=\mathrm{U}_{\mathrm{C} 2}=1.5 \mathrm{~V}$, a small well doping doze and small distance between the emitter and operating collectors. Relatively large operating collector current and maximum change of collector current in a magnetic field is observed. In this case, less recombination limits a current. The most part of the injected carriers reaches working collectors.
Fig. (5). represents the three-dimensional diagram of relative current sensitivity dependence versus the technological parameters defining the structure of the well. Relative current sensitivity characterizes internal change of a current in the device under influence of a magnetic field.

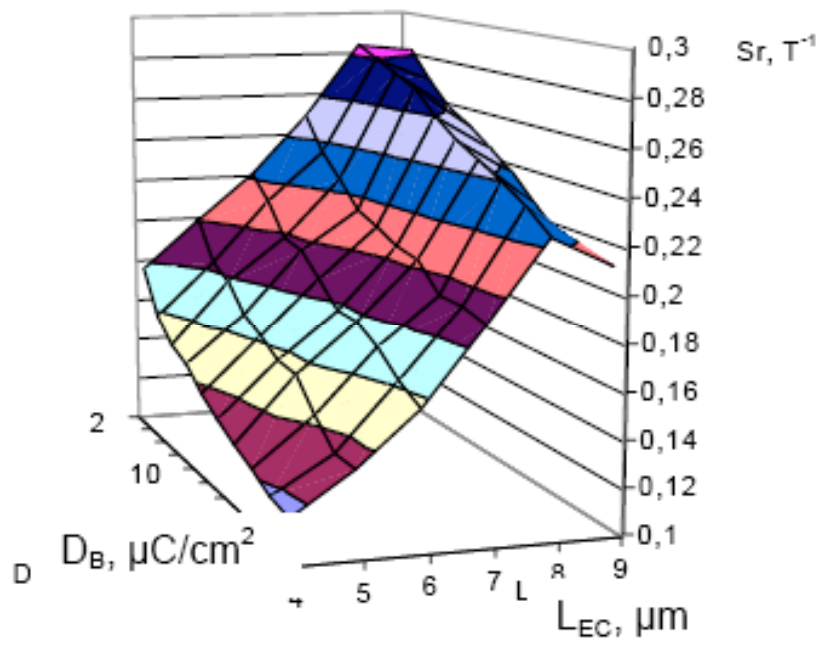

Fig. (5). Dependence of the relative current magnetosensitivity on the bias $0.72 \mathrm{~V}$ between the emitter and contacts to the well-base and the substrate in the bipolar dual-collector lateral magnetotransistor (BMT) about the boros dose $2-20 \mu \mathrm{C} / \mathrm{cm}^{2}$ and the emitter-collector distance $4-9 \mu \mathrm{m}$.

The current sensitivity is defined on change of a difference of a current of collectors Ic1-Ic2 in a magnetic field with an induction $\boldsymbol{B}$

$S_{R I}=(I c 1-I c 2) / B(I c 1+I c 2)$

The maximum values of change of the collector current and of relative current sensitivity are observed given the smallest well doping doze $2 \mu \mathrm{C} / \mathrm{cm}^{2}$ and the greatest distance between the emitter and collectors is 8 microns. The deflection of the injected carriers at the longer base increases relative sensitivity.

Extrapolation to within $10 \%$ of dependence of relative sensitivity $S_{\boldsymbol{R}}$ from technological parameters of a well of length of base lateral transistor $\mathrm{L}_{\mathrm{EC}}$ in a micron, a base doping doze $\mathrm{D}_{\mathrm{W}}$ at an identical termo mode gives the following formula:

$S_{R I}=0,067\left(L_{E C}^{0,75}-\lg D_{B}\right)$

From this formula follows, that relative sensitivity BMTW grows almost proportionally to length of base lateral transistor $\mathrm{L}_{\mathrm{EC}}$, at least up to some value of length of the base, 8 microns equal in this case. Change ionic doping bases poorly influences sensitivity, but all the same sensitivity above at weak doping bases doze $\mathrm{D}_{\mathrm{W}}=2$, instead of 20 $\mu \mathrm{C} / \mathrm{cm}^{2}$. It is necessary to note distinction of dependences on Fig. 4 and 5. Absolute sensitivity grows at reduction of length of base, and relative sensitivity grows at increase in length of base.

The knowledge of dependences of relative and absolute current sensitivity allows choosing the BMT geometry with the maximal sensitivity, i.e. to perform the sensitivity optimization of the device structure. At the chosen values of the well doping level, the maximum value of the relative current 
sensitivity increases by 2.5 times from $0,115 \mathrm{~T}^{-1}$ to $0,285 \mathrm{~T}^{-1}$. This result will be coordinated with data resulted in work [35] where at increase in distance between the emitter and collectors with 5 up to 25 microns in magnetotransistor with restriction of lateral injection relative sensitivity raised with $0,09 \mathrm{~T}^{-1} 1$ up to $0,5 \mathrm{~T}^{-1}$.

Comparison of BMTW with bottom and top contact to a substrate at device-technological modeling provides useful hints for the understanding of the mechanism of negative sensitivity occurrence of dual-collector magnetotransistors.

Calculated values of maximum relative current sensitivity at different positions of contacts to substrate produce values of $0,38 \mathrm{~T}^{-1}$ and 1,02/0,466 $\mathrm{T}^{-1}$, i.e. the bottom arrangement of contacts can give an increase in sensitivity. Absolute sensitivity experimental values of $1.8 \mathrm{~V} / \mathrm{T}$ for BMT, - $4 \mathrm{~V} / \mathrm{T}$ for BMTW with floating substrate, $-9 \mathrm{~V} / \mathrm{T}$ for BMTW with equal potentials at the base and at the substrate correspond to relative sensitivity of $0,4 \mathrm{~T}^{-1},-0,9 \mathrm{~T}^{-1},-2,8 \mathrm{~T}^{-1}$.

Possible increase of sensitivity due to the bottom arrangement of contact to the substrate requires the technology of double-side aligning. Advantages of planar technology allow us to consider the manufacturing of samples with bottom contact to be unpractical. The design of BMTW with associated base and substrate contacts and the design of BMTBW with base in the well [36-38] are more perspective.

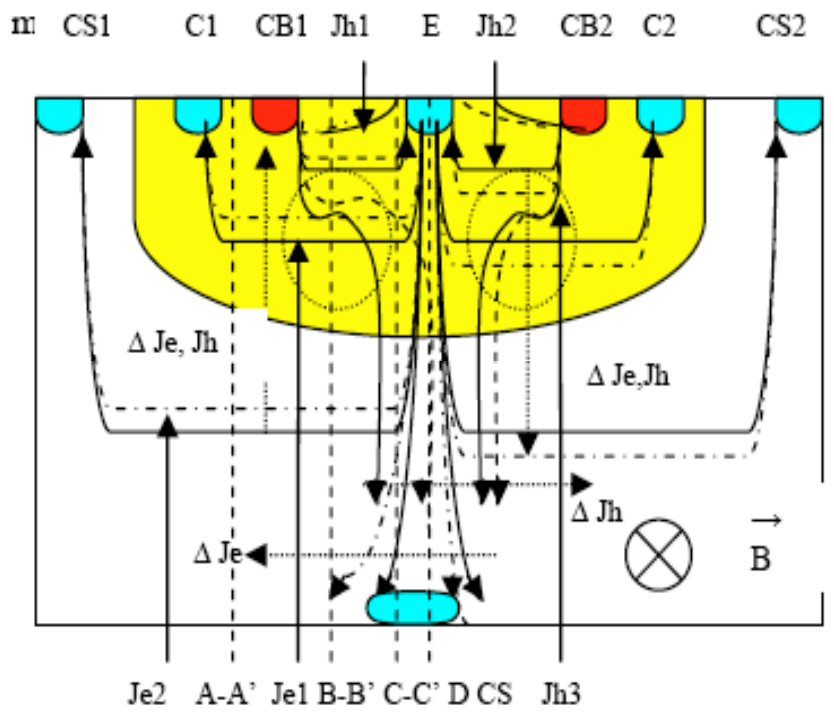

Fig. (6). Structure of dual collector lateral bipolar magnetotransistor, generated in the well. Conditional representation is of electron and hole current lines and the change of their configuration in a magnetic field.

\section{BMT STRUCTURE AND CURRENT-VOLTAGE CHARACTERISTICS}

With the view to studying the physics of BMT operation using a software package for the two-dimensional devicetechnological simulation ISE TCAD, a computational experiment has been carried out to compare two variants of the dual-collector lateral BMT generated in the well with equal potentials of contacts to the base-well and the substrate. In the first variant, the two contacts to the substrate are located on the upper side of the substrate. In the second variant, one contact is located on the bottom side of the substrate opposite of the emitter.

Fig. (6) shows the structure of the BMT with the $\mathrm{N}+$ emitter E, with two P+ contacts CB1, CB2 to P-diffusion base-well, with two $\mathrm{N}+$ collectors - $\mathrm{C} 1, \mathrm{C} 2$, with two $\mathrm{N}+$ contacts - CS1, CS2 to the upper side of the N substrate and with one contact CS below the substrate. The size of the elements horizontally: E - $4 \mu \mathrm{m}, \mathrm{CB} 1,2-20 \mu \mathrm{m}, \mathrm{C} 1,2-20$ $\mu \mathrm{m}, \mathrm{CS} 1,2$, CS $-20 \mu \mathrm{m}$. The length of the electrodes perpendicular the plane is $80 \mu \mathrm{m}$. The distance between elements E-CB1,2 - $20 \mu \mathrm{m}, \mathrm{CB} 1,2-\mathrm{C} 1,2-3 \mu \mathrm{m}$. Between the electrodes there is an oxide and under it - P-area of protection. Distribution of impurity for the cross section D taking place in the center of the structure is as follows. The Nsubstrate has a donor concentration of $10^{15} \mathrm{~cm}^{-3}$. In the basewell, the acceptor concentration decreases from the maximal value of $5 \times 10^{16} \mathrm{~cm}^{-3}$ near the emitter up to $10^{15} \mathrm{~cm}^{-3}$ at the junction well-substrate at the depth of $4.9 \mu \mathrm{m}$.

The electrical mode of BMT operation: the collector voltage $\mathrm{Uc} 1,2=1.5 \mathrm{~V}$, base and substrate voltage - Ube 0.45 $-1 \mathrm{~V}$. The magnetic field of $\mathrm{B}=1.81 \mathrm{~T}$ is directed from the observer parallel to the transistor surface. In Table 3, the currents of electrodes BMT result in the maximal negative sensitivity and Ube $=0,755 \mathrm{~V}$.

At the voltage $0.755 \mathrm{~V}$, the emitter current is 100 times as strong as the base current and practically all of the emitter current goes to the substrate. Currents of the measuring collectors are a million times less than the substrate current. It is the low injection mode. In this case, sensitivity is negative. At the voltage of $1 \mathrm{~V}$, the emitter current is only 5 times as strong as the base current, the emitter current is almost divided in half between the collectors and the substrate. It is the high injection mode. In this case, sensitivity is positive.

Fig. (7) shows the base-emitter current-voltage characteristics and relative sensitivity $S_{R I}$ in the maximal negative sensitivity area, calculated on the formula (7).

Before the operation threshold, with the emitter-base voltage Ube $=0.74 \mathrm{~V}$, the value of working collector cur-

Table 3. Current magnitudes for emitter Ie, base Ib, substrate Isub, first Ic1 and second Ic2 collectors inside the magnetic field $1,81 \mathrm{~T}$ and without a field $\mathrm{Ic1}(\mathrm{B})=\mathrm{Ic2}$, relative sensitivity under current $S_{\mathrm{RI}}$. Ube $=0,755 \mathrm{~V}$

\begin{tabular}{|c|c|c|c|c|c|c|c|}
\hline $\mathbf{I}, \boldsymbol{\mu} \mathbf{A}$ & Ie & Ib & Isub & Ic1 & Ic2 & $\begin{array}{c}\text { Ic1, Ic2 } \\
B=0\end{array}$ & $\mathbf{S}_{\mathbf{R I}}, \mathbf{T}^{-1}$ \\
\hline Down & 1063 & 12 & 1047,4 & 3,35 & 0,32 & 0,82 & $\begin{array}{c}1,02 / \\
0,466\end{array}$ \\
\hline
\end{tabular}


rents is less than $10^{-11} \mathrm{~A}$. An increase of the collector current results in the relative sensitivity accepting first negative, then positive values. The negative values of sensitivity exceed the positive ones.

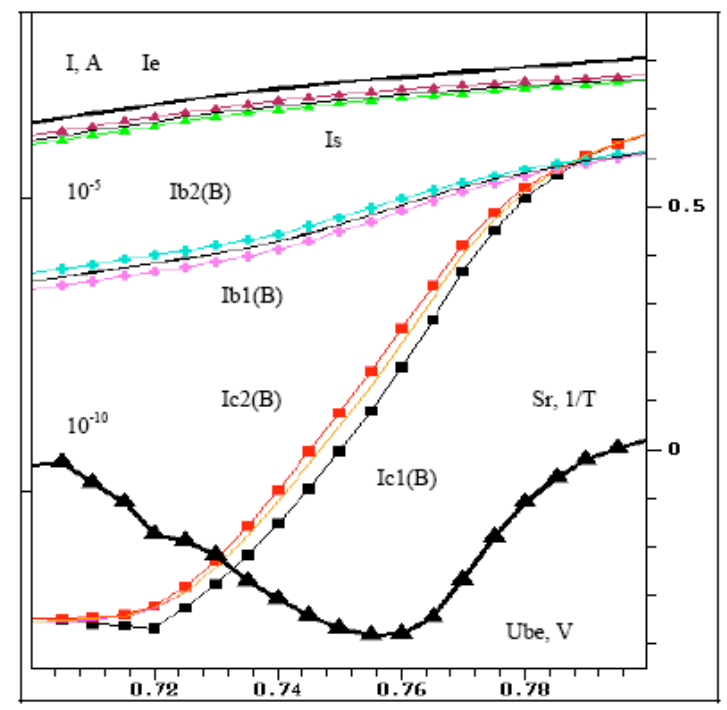

Fig. (7). Dependences of currents and sensitivity on top contact with the substrate.

\section{DISTRIBUTION OF CHARGE CARRIER CONCENTRATION, CURRENT DENSITY AND RECOMBINATION SPEED}

The concentration electrons injected from the emitter is shown in Fig. (8) for the maximal negative sensitivity mode (Ube $=0.755 \mathrm{~V})$ and is a little less than the hole concentration in the well near the emitter and much less in the direction of working collectors. It allows a conclusion that the device operates at low injection in some distance from the emitter towards the collectors and in a transitive mode of injection in the area near the emitter in the direction of the well-substrate transition, where the concentration of injected electrons is higher than that of holes in the well and that of electrons in the substrate. Recombination of charge carriers limits electron distribution along the surface and does not do so in the direction along the well-substrate junction.

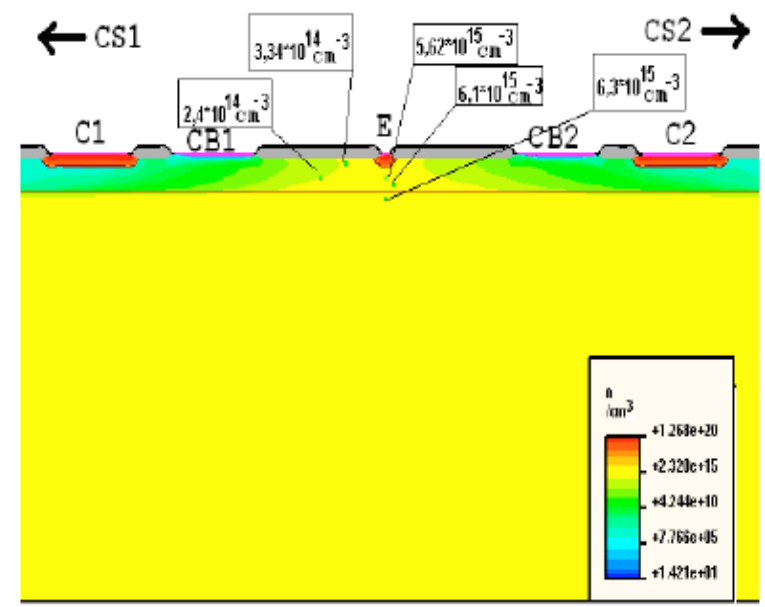

Fig. (8). The effect of the top contact with the substrate on distribution of electron concentration in the BMT.
The distribution of electron current lines directed opposite to the direction of the stream is given in Fig. (9) shows that in the low injection mode, current lines pass through the substrate from the contacts to the substrate to the emitter, only at the very emitter - through the well. A small number of electron current lines pass from the collectors through the well area. The magnetic field results in the asymmetry of current line arrangement. They move relative to the substrate surface. On the right, current lines in the substrate and the well move away from the surface and come close to the surface on the left. The vertical component of the electron stream from the emitter moves to the left in the substrate and the well.

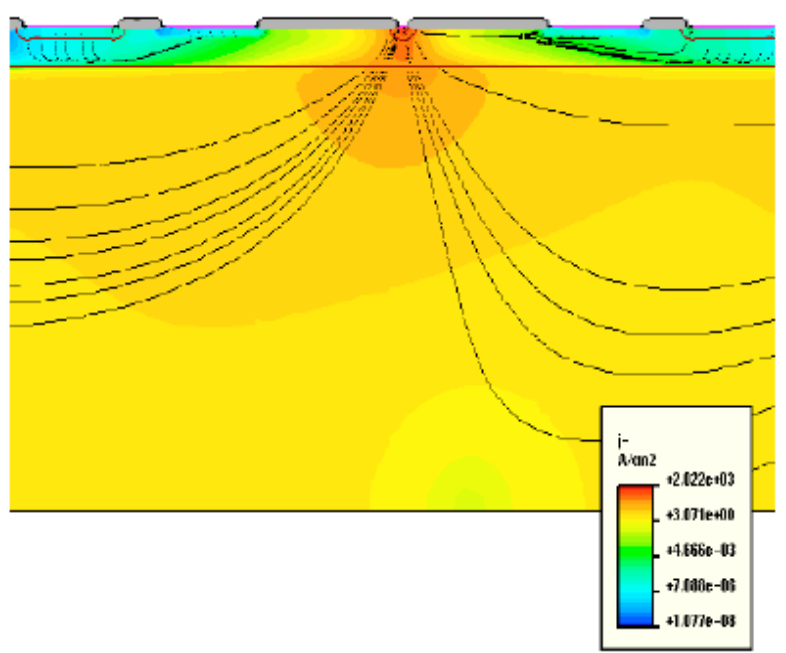

Fig. (9). The effect of the top contact with the substrate on distribution of electron current density and current lines in a magnetic field.

Fig. (10) makes it clear that hole current lines also change their location. Two hole streams going out of the contacts to the base are directed almost vertically. In the magnetic field, they both move to the right. There is a counter movement of electron and hole streams to the left of the emitter and in opposite directions to the right of the emitter. Opposite the emitter in the well-substrate junction area, there is a gap with a low hole current density. In the well near the well-substrate junction, the hole stream to the left of the emitter drifts towards the surface. To the right of it - the hole stream drifts away from a surface.

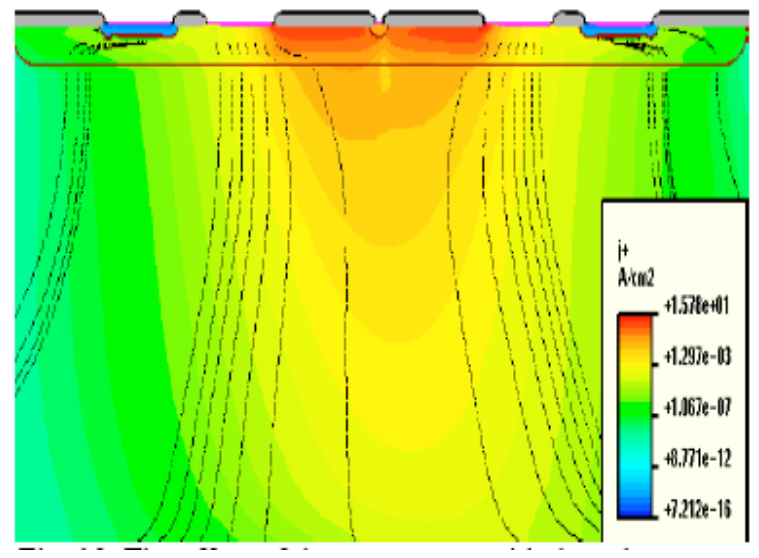

Fig. (10). The effect of the top contact with the substrate on distribution of hole current density and current lines in a magnetic field. 
At the well-substrate transition level, the distribution of electron and hole current density is given in Fig. $(\mathbf{1 1}, \mathbf{1 2})$. In Fig. (11), the electron current up to the left base contact edge in the magnetic field increases and exceeds the current without a magnetic field. The electron current up to the right base contact decreases.

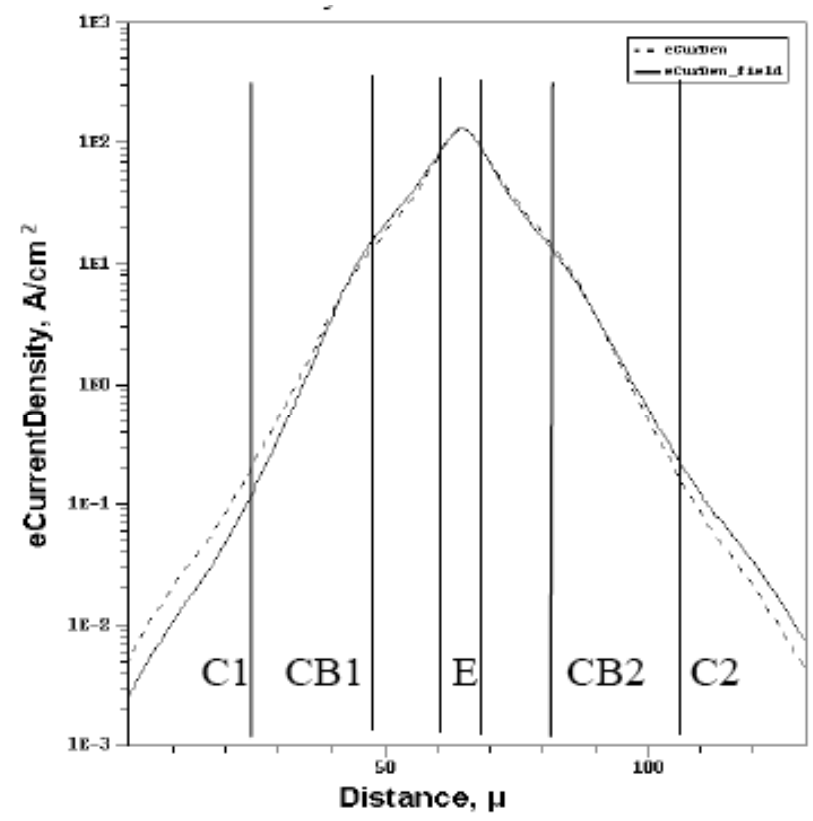

Fig. (11). The effect of the top contact with the substrate on distribution of density of an electron current flowing through the well substrate junction (-- without a magnetic field, _ in the field).

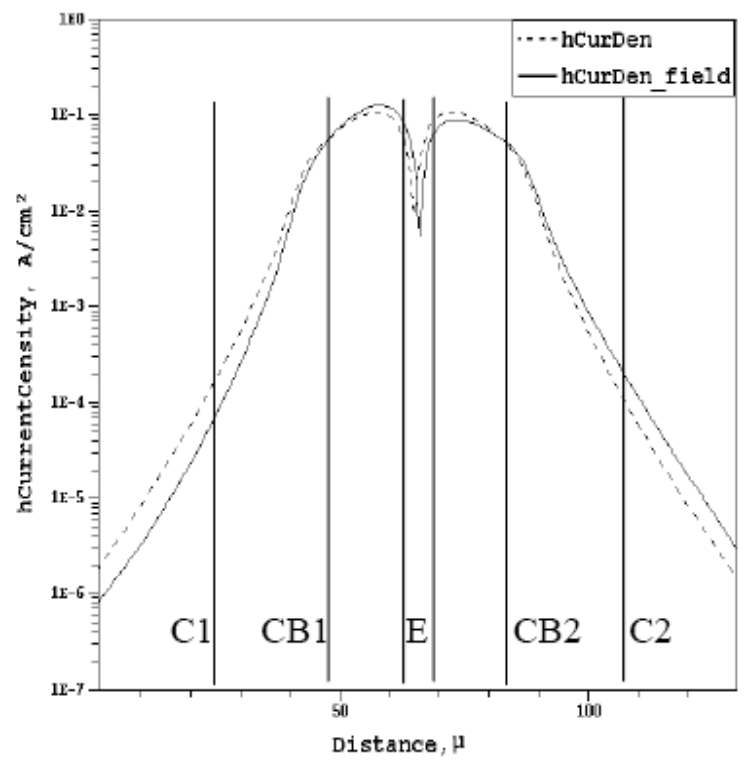

Fig. (12). The effect of the top contact with the substrate on distribution of a hole current flowing through the well - substrate junction (-- without a magnetic field, in the field).

The electron current opposite to the base contacts and farther to the left of the emitter decreases. To the right, it exceeds the current without a magnetic field. Up to the left base contact edge, the hole current, Fig. (12), in the magnetic field increases and exceeds the current without a magnetic field. Before the right contact in base, it decreases. Opposite the base contacts and farther on the left the hole current decreases and on the right, it exceeds the current without a magnetic field. Thus in the magnetic field, there occurs a redistribution of both electron and hole flows both simultaneously, i.e. passing of electron-hole plasma changes. As a result, currents to the left of the emitter to the substrate near the emitter increase and the working collector electron current decreases, as does the base hole current. To the right of the emitter, the current to the substrate near the emitter decreases and the working collector electron current, as well as the hole current of the base, increases. Nevertheless, the hole current emitter density falls down.

The above described variants of current distribution show that in the bipolar dual collector magnetotransistor structure, there occurs a galvanomagnetic effect - deflection of current lines. The magnetic field influences electron and hole currents with Lorentz force and results in a change of the distribution, configuration, and the effective length of current lines.

Injection changes concentration of charge carriers in some areas of the device. Due to the electro-neutrality condition, the change of electron and hole concentration is equal; the changes of distribution of electron concentration and hole concentration, therefore, also have the same character. In the well to the left of the emitter, in the area between the emitter and the contact to the base, there is an increase in both electron and hole concentrations, while on the right, their concentrations go down. Farther away from the emitter, closer to the contact to the base, concentration changes in an opposite way, i.e. it increases on the right and decreases on the left.

The change of charge carrier concentration distribution determines the magneto-concentration effect. In this BMT structure, the effect develops when there is no external field at the well-substrate transition point, while the diffusion transfer of injected charge carriers of both signs occurs, i.e. under the influence of a magnetic field on electron-hole plasma.

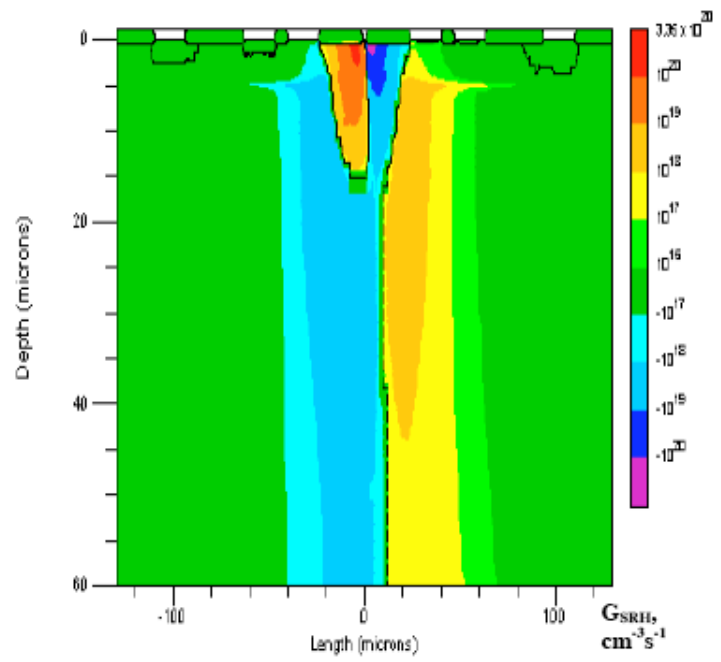

Fig. (13). The effect of the top contact with the substrate on distribution of a recombination speed difference according to Shockley-Read-Hall mechanism, in a magnetic field and without the field at Ube $=0.75 \mathrm{~V}$. 
The galvanomagnetic-recombination effect occurs in proportion to the electron and hole concentration change. In the magnetic field in the well and the substrate, near the well between the emitter and the contact to the base, the recombination speed grows to the left of the emitter and drops to the right. The change of recombination speed according to Shockley-Read-Hall mechanism in a magnetic field is shown in Fig. (13). In the vicinity of the contact to the base and in the area before the collectors, the effect has the opposite sign: the recombination speed decreases to the left of the emitter and grows to the right.

Intensified by the magnetoconcentration effect, the recombination of carriers in the area between the emitter and the base contact results in a decrease of the electron flow towards the left collector. The right collector electron flow density grows and therefore, the collector 2 current turns out to exceed the collector 1 current. This gives negative current sensitivity to the transistor.

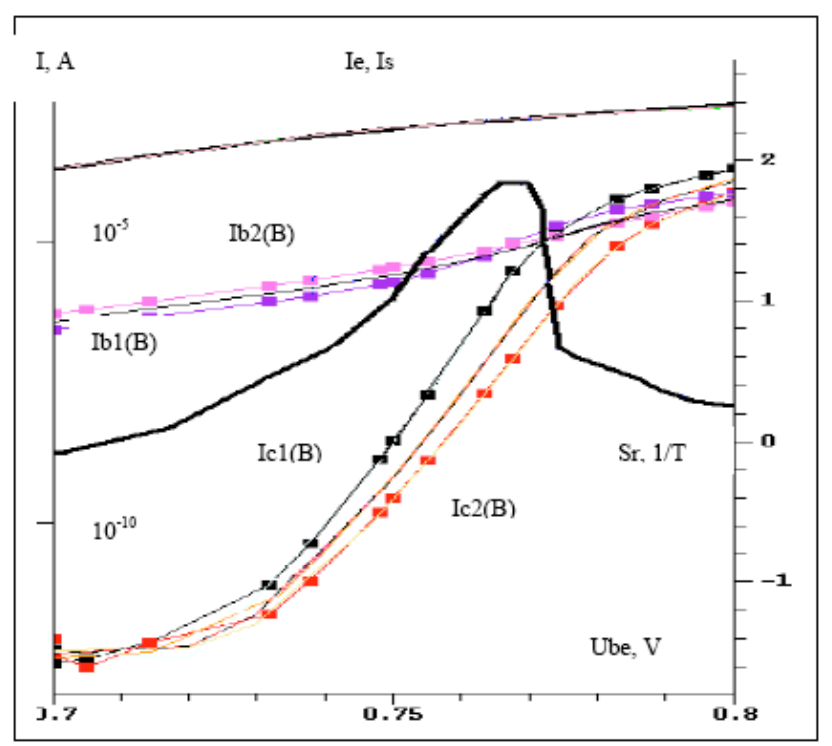

Fig. (14). The effect of the bottom contact with the substrate on current distribution and sensitivity.

If the substrate contact is placed on the bottom surface, negative sensitivity is not observed. Fig. (14) shows the base-emitter voltage dependence of current-voltage characteristics and relative sensitivity $S_{\mathrm{RI}}$ for the bottom arrangement of substrate contact in the field of bias voltage with maximal negative sensitivity for a BMT with top substrate contacts. Starting from the threshold of operation - the voltage at which the current of collectors begin to grow - collector 1 current exceeds collector 2 current.

The current distribution pattern changes as the substrate contact is repositioned. Electron flow lines mainly go vertically; therefore the magnitude of collector currents is reduced as compared to the structure with top substrate contacts at the same bias voltage.

At the well-substrate junction, the electron and hole current density distribution shows (Fig. 15, 16), that electron and hole currents in magnetic field increase up to the left base contact edge and to the collector, while they decrease up to the right base contact and to the collector. On the left, behind the base contact, electron current density in magnetic field is higher than that without a magnetic field. On the right behind the base contact it falls. The collector 1 current exceeds the collector 2 current, which corresponds to BMT's positive current sensitivity. The bottom arrangement of the substrate contact causes low electron current density in the well between the emitter and the base contact which determines an insignificant increase in the recombination speed in the well to the left of the emitter.Between the base contact and the collector appears additional current from the electron flow in the substrate outside the well. This additional current grows in a magnetic field, thus, producing a substantial effect on the collector current sensitivity sign.

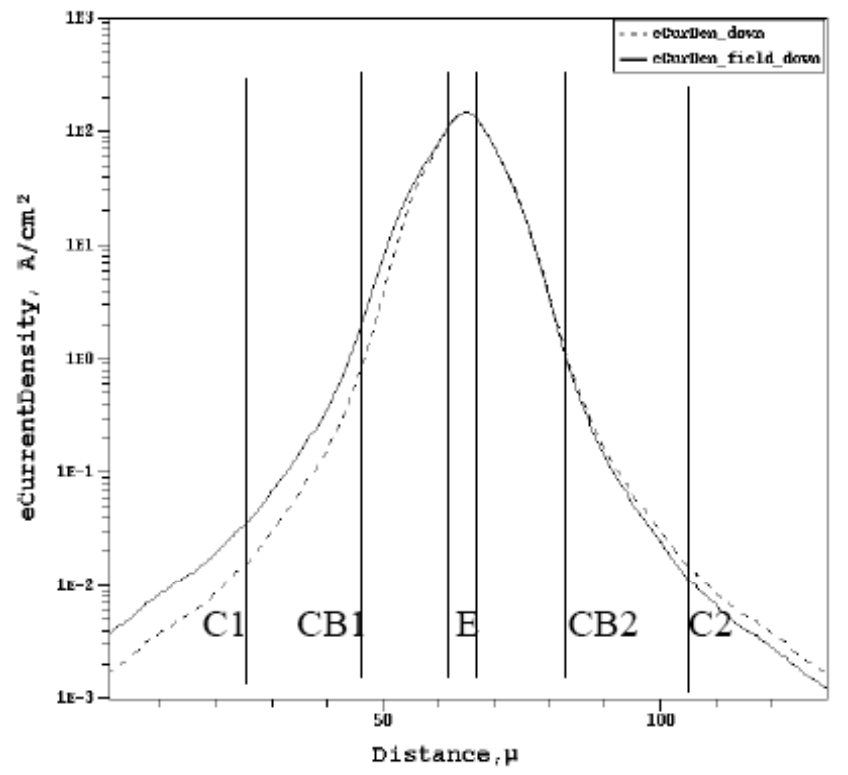

Fig. (15). The effect of the bottom contact with the substrate on distribution of density of an electron current flowing through the well - substrate junction (-- without a magnetic field, in the field).

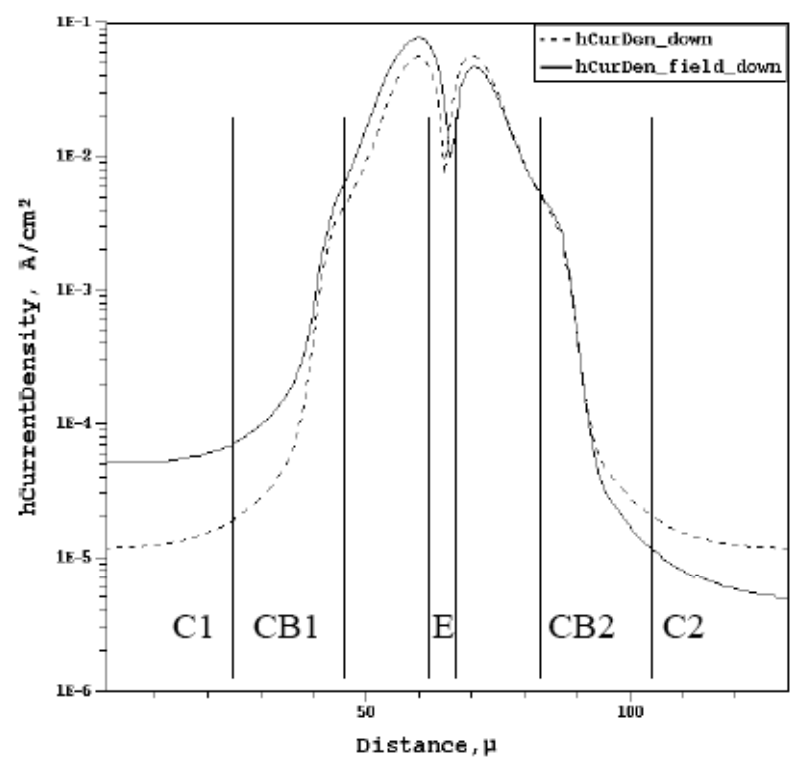

Fig. (16). The effect of the bottom contact with the substrate on distribution of density of a hole current flowing through the well substrate junction (-- without a magnetic field, in the field). 
As it is clear from Fig. (17), in the device there occurs a change of recombination speed, which is in proportion to the increase of electron and hole concentration in the well to the left of the emitter, between the emitter and the contact to the base, and the decrease to the left of it.

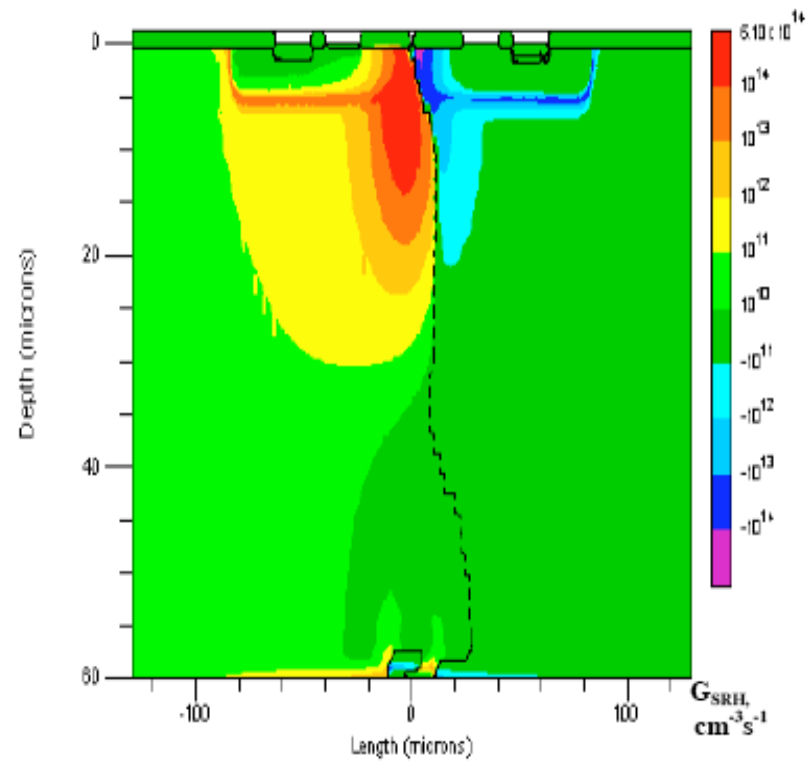

Fig. (17). The effect of the bottom contact with the substrate on distribution of a recombination speed difference according to Shockley-Read-Hall mechanism in a magnetic field and without the field at Ube $=0.75 \mathrm{~V}$.

\section{DISCUSSION OF THE MODELING RESULTS}

More exact comparison of distribution of density of an electronic current in a well on section B-B' between the emitter and contact to base and section A-A' between contact to base and a collector in Fig. (6) are presented in a Fig. $(\mathbf{1 8 , 1 9 )}$ for both variants of structure BMT and in an identical operating mode.

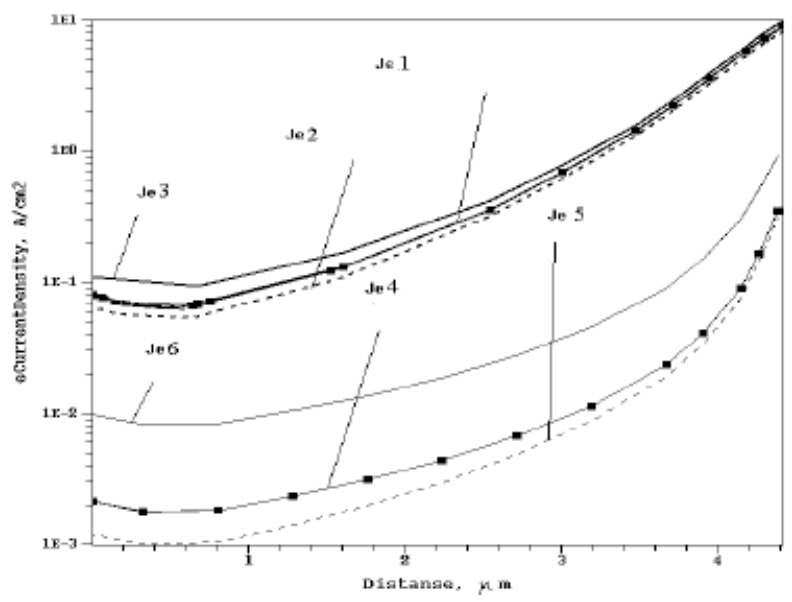

Fig. (18). Density of an electronic current between the emitter and contact to base at the top and bottom contacts to a substrate in section B-B'.

On the Fig. (18) between the emitter and the contact to the base the electron current density is 35 times as high for the structure with top contacts to the substrate (Je1) as that for the bottom contact arrangement (Je4). In the vicinity of the well-substrate junction, the injected electron current density, in the case of both top and at bottom contacts, is 100 times as high as that near the surface of the device. If there is a magnetic field, according to the Lorentz force vector, density grows by $50 \%$ in the left section for the top-contacts structure (Je3) and 5-fold for the bottom-contacts structure (Je6), i.e. in case of bottom contacts the relative change of current density is 10 times higher. To the right of the emitter in section B-B' density decreases (Je2, Je5).

In section A-A', between the contact to the base and the collector, electron current density (Fig. 19) is 16 times higher in the top-contacts structure (Je1) than in the bottomcontacts one (Je4). In the vicinity of the well-substrate junction current density is 1000 times higher than near the surface both with top and bottom contacts. In comparison with section B-B', electron current density decreases 100-fold near the well-substrate junction and 1000-fold on the surface, i.e. the flow of current along the surface is strongly limited by recombination at high concentrations impurity in the base-well near the surface. The section A-A'/section BB' density ratio decreases twice for top and bottom contacts. The significant current flow through the base-well area in the case of top contacts to the substrate is limited by recombination to a larger extent than the smaller current flow in the case bottom arrangement of the contact to the substrate.

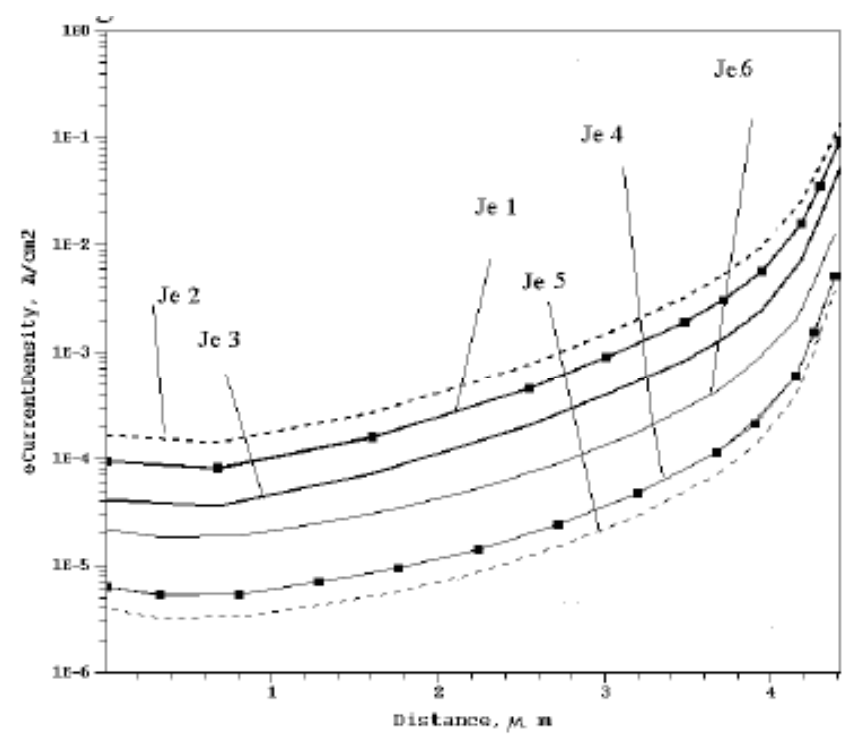

Fig. (19). Density of an electronic current between the emitter and contact to base at the top and bottom contacts to a substrate in section A-A'.

For the bottom-contact structure with a magnetic field in the section A-A', electron current density in the left section grows 3 times (Je6). In the right section, it decreases 1.5 times according to the direction of Lorentz force (Je5). For the top-contact structure with a magnetic field, density in the left section falls twice (Je3) while in right section, it grows 1.5 times (Je2) opposite the direction of Lorentz force !!!.

The top arrangement of contacts to the substrate is characterized by a significant component of the electron current flowing through the well areas with a high impurity concentration. To the left of the emitter, this component grows un- 
der the influence of a magnetic field produced on the stream of injected electrons because of the galvanomagnetic effect of deflection. At the same time, current lines move to the base area near the surface with a higher impurity concentration and a higher recombination speed. The recombination speed grows so much that at a certain distance from the emitter, current density falls and fewer electrons reach the collector than would be the case without a magnetic field. To the right of the emitter these effects have the opposite sign.

This makes it possible to state that the electron current distribution in the BMT base plays an important role in the mechanism of operation. The presence of a significant electron current component in the base-well with the top arrangement of contacts to the substrate results in a negative sensitivity. The bottom arrangement has no negative sensitivity despite the complete identity of all the other BMT structure elements.

The hole current density distribution in the sections B-B' (Fig. 20), C-C' (Fig. 21) shows that in the upper part of the base this distribution corresponds to the acceptor distribution in the base, i.e. the hole current in the base at a low level of electron injection is determined by a drift of impurity conductivity holes.

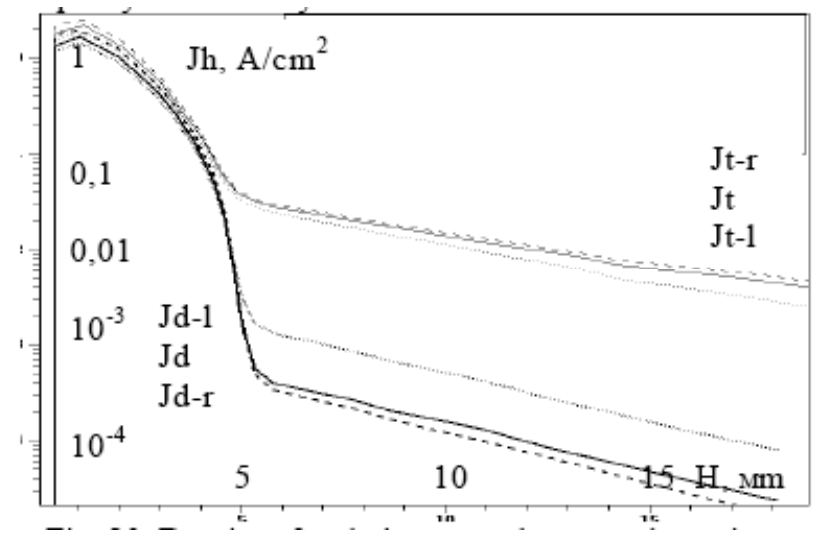

Fig. (20). Density of an hole current between the emitter and contact to base at the top and bottom contacts to a substrate in section B-B'(20 $\mu \mathrm{m}$ from centre).

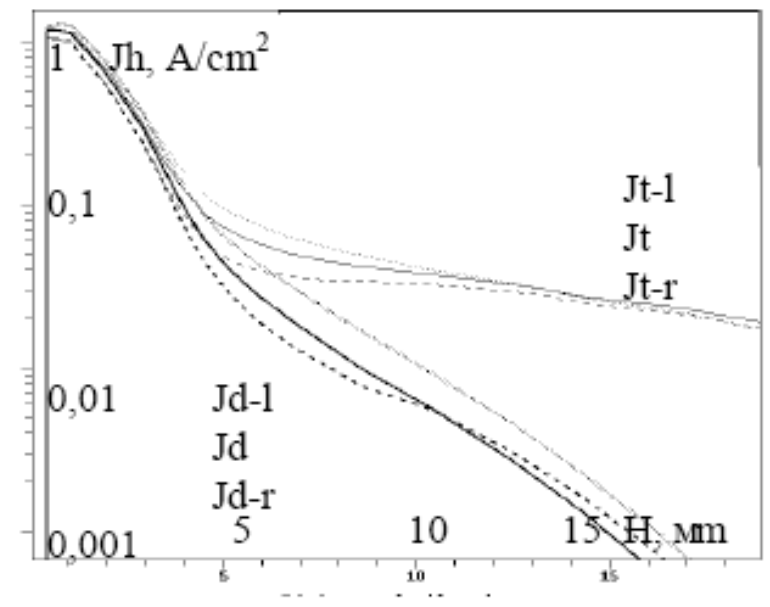

Fig. (21). Density of an hole current between the emitter and contact to base at the top and bottom contacts to a substrate in section C-C'( $3 \mu \mathrm{m}$ from centre).
In the B-B' cross section, the current density is 1.5 times higher than in the C-C' cross section. In the space between cross sections, the reduction of hole current density takes place at the expense of several factors as shown in Fig. (6). One part of hole current Jh2 entering the recombination with electrons in the base and on the base-well surface decreases while moving away from the base contact. The other part of the hole current Jh1, almost identical in two structures, passes into the emitter and results in electron injection. In the region of intensive injected electron flow that carries away holes downwards into the substrate, there is hole current $\mathrm{Jh} 3$, opposite in direction, that flows to the emitter from the bottom up. Meeting currents compensate each other, creating the reduction of hole current density in the base opposite to the emitter. As it is shown in Fig. (6), Jh3 hole current flows together with electrons into the substrate and recombine there.

In section B-B' at the top arrangement of contacts to a substrate, density of a hole current in base $\mathrm{Jt}$ is $30 \%$ more Jd. In section C-C' the distribution of hole currents in a well coincides in two structures $\mathrm{Jt}=\mathrm{Jd}$ and the difference is present only in a substrate on the large depth Jt $>\mathrm{Jd}$. Density of hole current $\mathrm{Jd}<\mathrm{Jt}$ in section B-B' is 100 times more near to the junction well-substrate and in a substrate than at the bottom position of contact. On a condition of electroneutrality, the large hole current in a substrate near the base contact is determined by an electron flow through base in a substrate at the top placement of contacts to a substrate.

For both structures in a magnetic field in the base-well to the left of the emitter near the contact to the base in the section B-B', the hole current density Jt-1, Jd-1 falls, while growing to the right of it Jt-r, Jd-r. In the substrate, the magnetic field creates a similar effect for the top arrangement of contacts to the substrate: to the left of the emitter near the contact to the base in the section B-B', the hole current density decreases, while increasing to the right of it. For the bottom-contact structure, an opposite effect is observed closer to the substrate: the hole current density in the substrate grows to the left of the emitter, though remaining much lower than in the top-contacts structure, and falls to the right of the emitter.

In section $\mathrm{C}-\mathrm{C}^{\prime}$ about the emitter, the magnetic field reduces density of a current on the right and increases at the left in a well as well as in a substrate at two variants of an arrangement of contacts to a substrate. The increase of density of a hole current about the emitter at the left results in increase of recombination speed. On the right, reduction of a hole current is accompanied by reduction of recombination speed (Fig. 17).

In the section $\mathrm{C}-\mathrm{C}^{\prime}$ near the emitter, the magnetic field reduces current density on the right and increases on the left in the well and substrate for both variants of contact arrangement. The increase of hole current density near the emitter on the left results in a higher recombination speed. On the right, the hole current reduction is accompanied by a lower recombination speed (Fig. 17).

The change of hole concentration near the contact to the base at a given base voltage down the hole current through the left contact to the base and pushes up the current of the right contact (Fig. 6). Base resistance grows on the left and 
falls on the right. This galvanomagnetic effect can be classified as the Gauss effect - base resistance growing in a magnetic field. The calculations of mobility of carriers show that at a given magnetic field value change is less than $1 \%$. The change of current lines length contributes most of all.

The magnetic field affects three hole current components in base Jh 1,2,3 shown in Fig. (6). The recombination component in base Jh1 should grow to the left of the emitter and fall to the right due to changing recombination speed in the vicinity of the emitter (Fig. 13). To the left of the emitter, electron and hole currents keep closer to the surface, where impurity concentration is higher. On the right, they flow away from the surface toward the well-substrate transition, where the impurity concentration is lower. The bias current of the Jh2 emitter junction flows along the surface in the area of strong enough base doping and changes little in a magnetic field. For these two current components, the effects are similar in both variants of contact arrangement.

The difference between these two structures is observed in the area between the contact to the base and the emitter on the side of the contact to the base (marked with an oval in Fig. 6), where in the top-contacts structure, mostly injection electron current flows in the substrate together with the hole current compensating charge. In the top-contacts structure, closer to the substrate the hole current going for recombination to the substrate, Jh3 grows in a magnetic field and passes with a bias to the right, i.e. along a longer path, which determines an increase of base resistance to the left of the emitter. To the right of the emitter, the length of hole current lines decreases and the resistance falls.

\section{CONCLUSIONS}

Negative sensitivity of a dual-collector lateral magnetotransistor generated in a diffused well in comparison with a positive sensitivity magnetotransistor generated in a uniformly doped substrate was experimentally established. Comparative analysis of sensitivity of all semiconductor devices makes it possible to introduce the concept of sensitivity sign and classify the sensitivity of a dual-collector lateral magnetotransistor generated in a diffused well on the basis of the chosen criterion.

Device- technological simulation was used to study the mechanism of occurrence of different sensitivity polarity in view of Galvanomagnetic effects.

Earlier the effect of structural dependence of a sign on sensitivity bipolar magnetotransistor is not described by anybody.

The comparison of the current density distribution in the base-well for two BMT variants of an identical structure but with a different arrangement of contacts to the substrate shows that the physical galvanomagnetic concentrationrecombination effect occurs. In a magnetic field, current lines of basic and injection charge carriers change their position and configuration. The concentration of carriers and recombination speed also change in different areas of the BMT. The impurity concentration and current distribution is determined by the structure of the two-collector lateral BMT generated in the well and operating with equal potentials at the contacts to the base and the substrate. Galvanomagnetic effects create a concentration-recombination mechanism of
BMT negative sensitivity. Understanding of the sensitivity mechanism allows developing designs of magnetic sensors with high sensitivity.

\section{REFERENCES}

[1] H. Weiss, "Physik und Anwendung Galvanomagnetischer Bauelemente" F. Vieweg und Sohn, Braunschweig, 1969.

[2] I.S. Levitas, Yu.K. Pojela, A.P. Saschuk, "Investigation of the kinetic of the Galvanomagneticrecombination effect" Sov. Phys. Semicond, p. 205, 1972.

[3] Malytenko V.K., Bolgov S.S., Malozovsky Yu. M., "Magnetoconcentration effect at nonlinear recombination of current carriers" Phys. Stat. Sol.(a), p. 723, 1978.

[4] S. Cristoloveanu, "Magnetic field and surface influences on double injection phenomena in semiconductors. Part 1// Phys. St. Sol., , p. 683,. 1981, Part 2, p. 281, 1981.

[5] I.M. Vikulin, V.I. Stafeev, "Physics of Semiconductor Devices" Moscow, RF, Radio and Svijs, 1990.

[6] E.C. Hudson Jr., "Semiconductive magnetic transducer" Patent US 3,389,230, 1968 .

[7] I.M. Mitnikova, T.V. Persiyanov, G.I. Rekalova, G. Shtyubner, "Investigation of the characteristics of silicon lateral magnetotransistors with two measuring collectors' Sov. Phys. Semic, p.26, 1978.

[8] H.P Baltes, R.S. Popovic, "Integrated semiconductor magnetic field sensors" Proc. IEEE, p. 1107, 1986.

[9] C.S. Roumenin, "Bipolar magnetotransistor Sensors. An invited review" Sensors and Actuators A, p. 83, 1990.

[10] R.S. Popovic, R. Widmer, "Magnetotransistor in CMOS technology" IEEE Trans. Electron Divices, p.568, 1986.

[11] A.W.Vinal, N.A. Masnari, "Operation principle of bipolar transistor magnetic sensors” IEEE Trans. Electron Dev., p. 1486, 1984.

[12] L.J. Ristic, H.P. Baltes, T. Smy, I. Filanovsky, "Suppressed sidewall injection magnetotransistor with focused emitter injection and carrier double deflection" IEEE Electron Divice letters, p.395, 1987.

[13] Y.G. Song, J.G. Ryu, Y.S. Choi, N.H. Kim, "Fabrication and characteristics of the suppressed sidewall injection magnetotransistor using a CMOS process" Sensors and Actuators A, Physical, p. 99, 2006.

[14] C. Riccobene, G. Wachutka, J. Burgler, H. Baltes, "Operation principle of dual collector magnetotransistors stadied by twodimensional simulation” IEEE Trans. Electron Dev., p. 1136, 1994.

[15] R. D. Tikhonov, "Sign magnetosensitivity of dual-collector lateral bipolar magnetotransistor" The International Conference, "Microand nanoelectronics - 2007" Report O1-30, Zvenigorod, Russia,15.10.2007.

[16] E.V. Kuchis, "Galvanomagnetic Effects and metods of investigation" Moscow, RF, Radio and Svijs, 1990.

[17] G.A. Egiazarian, V.I. Stafeev, "Magnetodiodes, magnetotransistors and thear aplication" Radio and Sviyz; Moscow, RF, 1987.

[18] L.W. Davies, M.S. Wells, "Magneto-transistor incorporated in an IC” Proc. IREE, Australia, p.235, 1971.

[19] I.M. Vikulin, M.A. Glauberman, L.F. Vikulina, Yu. I. Zaporozhchenko, "Investigation of the characteristics of a twocollector magnetotransistor” Sov. Phys. Semicond., p. 369, 1974.

[20] C.S. Roumenin, "Optimization Bipolar magnetotransistor Sensors" Sensors and Actuators A, p. 83, 1990.

[21] R.D. Tikhonov, "Mechanisms Sensitivity of a Bipolar Magnetotransistor ch.1" Measurement Techn., p.39, 2006.

[22] V.V. Amelichev, A.I. Galushkov, Yu.N. Mirgorodskii, P.A. Tichomirov, Yu.A. Chaplygin, M.V. Shorin, S.V. Shubin, "Modeling bipolar two-collector magnetotransistors and judgement for termocompensation sensitivity" Sensor and Systems RF, p. 38, 1999.

[23] M.A. Korolev, Yu.A. Chaplygin, V.V. Amelichev, R.D. Tikhonov, M.V. Shorin, "An investigation of the opportunity of bipolar lateral magnetotransistor sensitivity enhancement" News of Institutes of Higher Education. Electronics, p. 40, 2002.

[24] A.V. Kozlov, M.A. Korolev, S.Y. Smirnov, Yu.A.Chaplygin, R. D. Tikhonov, "Triple-Collector Lateral Bipolar Magnetotransistor: Response Mechanism and Relative Sensitivity" Russian Microelectronics, p. 172, 2003.

[25] A.V. Kozlov, M.A. Reveleva, R.D. Tikhonov, "The investigation of the negative sensitivity of the bipolar magnitosensitivity transis- 
tor" News of Institutes of Higher Education. Electronics, p.57, 2003.

[26] A.V. Kozlov, M.A. Reveleva, R.D. Tikhonov, "The investigation of the volume recombination of the bipolar magnitosensitivity transistor" Sensors and systems RF, p. 24, 2003.

[27] A.V. Kozlov, M.A. Reveleva, R. D. Tikhonov, "Dual-Collector Lateral Bipolar Magnetotransistor: Carrier Transport and Relative Sensitivity" Russian Microelectronics, p. 385, 2003.

[28] R.D. Tikhonov, "Concentration-recombination Bipolar Magnetotransistor: Response Mechanism and Relative Sensitivity" Russian Microelectronics, p. 382, 2004.

[29] A.V. Kozlov, R.D. Tikhonov, "Concentration-recombination Sensitivity Magnetotransistor" Sensors and systems RF, p. 40, 2004.

[30] R.D. Tikhonov, "Investigation of bipolar magnetotransistor" Proceedings of SPIE, vol. 6260, Micro- and Nanoelectronica 2005, p. 443, 2006.

[31] R.D. Tikhonov, "Mechanisms Sensitivity of a Bipolar Magnetotransistor ch. 2" Measurement Techn., p. 44, 2007.
[32]

R.D. Tikhonov, "Measuring Dual-Collector Lateral Bipolar Magnetotransistor with Base in Well: Negative Sensitivity and Noises" Measurement Techn., p. 47, 2007.

[33] Ch.S. Roumenin, "Solid State Magnetic Sensors", ELSEVIER SCIENCE, Amsterdam-Lausanne-New York-Oxford-ShannonTokyo, 1994

[34] A. Kozlov, M. Reveleva, R. Tikhonov, "The optimisation of relative current sensitivity of bipolar magnitotransistor" Proceedings of SPIE, vol. 5401, p. 362, 2004

[35] M. Metz, "Diss:Offset in CMOS Magnetotransistors, Analysis and Reduction" Zurich, 1999.

[36] R.D. Tikhonov, "Raising the Sensitivity of a Bipolar Magnetotransistor" Measurement Techn., p. 186, 2005.

[37] R. D. Tikhonov, "Response Mechanism of the Base-in-Well Bipolar Magnetotransistor sensitivity" Russian Microelectronics, p. 382, 2005.

[38] R. D. Tikhonov, "Sensor on Bipolar Magnetotransistor with Base in Well" Solid State Electronics, p. 1302, 2005.

(C) R.D. Tikhonov; Licensee Bentham Open.

This is an open access article distributed under the terms of the Creative Commons Attribution License (http://creativecommons.org/license/by/2.5/), which permits unrestrictive use, distribution, and reproduction in any medium, provided the original work is properly cited. 